\title{
Human Emergency Behaviour Characteristics in Large-scale Emergencies Based on Mobile Communication Data
}

\section{XiaoLin Wei}

Zhejiang Yuexiu University

Yi Luo ( $\nabla$ yluo@stu.zyufl.edu.cn)

Zhejiang Yuexiu University of Foreign Languages https://orcid.org/0000-0003-4367-8535

\section{ZhenZhen Chen}

Zhejiang Yuexiu University of Foreign Languages

\section{XuMing Zhao}

Zhejiang Yuexiu University of Foreign Languages

\section{HongYan Li}

Zhejiang Yuexiu University of Foreign Languages

\section{Tao Wang}

Shaoxing University

\section{Wei Yu}

Zhejiang Yuexiu University of Foreign Languages

\section{Lin Zhu}

Zhejiang University of Science and Technology

\section{FangYuan Liu}

UCSI: UCSI University

\section{Research Article}

Keywords: Mobile Communication Data, Large-scale Emergencies, Human Emergency Behaviour Characteristics, Time Series Analysis, Data Mining

Posted Date: December 22nd, 2021

DOI: https://doi.org/10.21203/rs.3.rs-1177241/v1

License: (c) (1) This work is licensed under a Creative Commons Attribution 4.0 International License.

Read Full License 


\title{
Human emergency behaviour characteristics in large-scale emergencies based on mobile communication data
}

\author{
XiaoLin Wei ${ }^{1}$, Yi Luo ${ }^{2,3^{*}}$, ZhenZhen Chen ${ }^{2,3^{*}}$, XuMing Zhao ${ }^{4}$, HongYan Li ${ }^{2,3}$, Tao Wang ${ }^{5}$, Wei Yu ${ }^{2,3}$, \\ Lin $\mathrm{Zhu}^{2,6}$ and FangYuan $\mathrm{Liu}^{7}$
}

\footnotetext{
${ }^{*}$ Correspondence:

yluo@stu.zyufl.edu.cn;

20192057@zyufl.edu.cn

${ }^{1}$ Zhejiang Yuexiu University ,

Shaoxing, China

Full list of author information is

available at the end of the article
}

\begin{abstract}
The characteristics of human emergency behaviour under the emergency are a crucial scientific issue in basic emergency management research. The analysis of time dynamic aspects of human behaviour based on electronic footprint data provides a new method for quantitative investigation of this problem. Previous studies generally assumed that human behaviours were randomly distributed in time, but few studies studied the impact of emergencies and carried out prediction methods through social media data. Using mobile $Q Q$ space communication data, this paper from four kinds of emergencies and one kind of conventional event data, digging out the statistical characteristic on the time dimension of human communication behaviour, and in case of any emergencies, such as public security mode of evolution, to explore intrinsic emergency regularity of the impact of human communication behaviour model and further predict human behaviour characteristics. We found that the communication peaks accompanying an emergency are local in time, resulting in a communication avalanche that importantly engages eyewitness social networks. In mobile $Q Q$ space communication, the probability distribution of the interval time of the Posting behaviour sequence shows the statistical characteristics of power-law and approximate exponential tail. Compared with most of the typical Posting behaviour, the probability distribution of the interval time of the Posting behaviour sequence is higher. At the same time, the mnemonic is lower than most of the typical Posting behaviour, with a weak anti- mnemonic. These results are theoretically helpful in understanding the regularity of the impact of emergencies on human communication behaviour patterns and have potential application value in predicting the impact degree of crises and the analysis and classification of human social attributes.
\end{abstract}

Keywords: Mobile Communication Data; Large-scale Emergencies; Human Emergency Behaviour Characteristics; Time Series Analysis; Data Mining

\section{Content}

\section{Introduction}

A series of human behaviours, such as communication, travel, shopping and socializing, is the direct or indirect driving forces of the typical complex system of human society. The academic circle has widely discussed how to deeply understand the regularity and internal driving mechanism of the human micro-behaviour pattern at the macro level and then apply it to the control of the social system. At present, statistical physics methods in big data make this idea possible. In 2009, Baraba Si's 
complex Systems and Networks album [1], published in The journal Science, predicted the next decade of complexity research. The paper proposes: "From mobile phones to GLOBAL positioning systems and the Internet, there is a rapid increase in the number of electronic devices in daily use that capture everything from our communications to our location. So the first complex system that we're most likely actually to quantify is probably not a cell or the Internet, but human society." At the same time, 15 internationally renowned scholars headed by Professor Lazer, including Baraba'si, jointly published a paper entitled "SOCIAL SCIENCE: The Computational Social Science "article[2] marks the birth of an interdisciplinary field of data-driven Computational sociology, which is based on statistical physics and focuses on the study of temporal and spatial dynamics of human behaviour, the characteristics and evolution of Social networks, data access and privacy protection, and the cultivation of new academic mechanisms. Subsequently, Castellano et al. [3], Conte et al. [4], Pentland[5] also reviewed the research issues and methods in this field. The dynamics of human behaviour, initiated by statistical physicists, have become a branch of computational sociology. Human behaviour is to study the characteristics and rules of human behaviour in time, space and social complexity through quantitative statistics of individual human behaviour and group behaviour. To reveal hidden statistical laws, explore their mechanisms and their impact on social, economic, technological and ecological external systems. It was generally assumed that human behaviour occurred randomly in time before this. The sequence of behaviours was approximately a Poisson process with an exponential distribution of intervals or waiting times.

However, Baraba'si's research on non-Poisson characteristics of time distribution of human activities and interpretation work based on priority queuing mechanism in 2005 [6], and Brockmann et al. 's quantitative research on spatial scaling law of human movement in 2006 [7] were successively published in Nature. It has become a historical turning point in human behaviour's temporal and spatial complexity analysis. At present, the study of human behaviour dynamics has become a hotspot of complexity science research in the world. More than 30 papers have been published in top international academic journals, such as Nature [8], Science [9] and Proceedings of the National Academy of Sciences[10]. A series of representative related work has also been published in Physical Review Letters[10], Europhysics Letters [11], Physical Review E [12], New Journal of Physics [13] and other Physics journals have been published. Meanwhile, relevant research work has also been published in IEEE Pervasive Computing[13], ACM UbiComp[11], SIGKDD[14], SIGSPATIAL GIS[15] and other international journals and conferences in the field of computers. It involves empirical analysis, theoretical modelling and application of time and space characteristics of human behaviour.

The research progress of human behaviour dynamics is mainly focused on studying human routine behaviour patterns. At the same time, more and more scholars began to focus on quantitative analysis of human behaviour patterns under the influence of emergencies based on large-scale human electronic footprint data. They applied it in the field of emergency management [16]. With the development of the Internet, people are constantly active in cyberspace, and human behaviour patterns can be expressed from the side of human social media communication behaviours. 
With the deepening of social interaction, many posts and comments generated by social media allow the study of the law of human behaviour and reveal the direction of human behaviour more accurately. In the monopoly era of mobile Qzone communication, the data of mobile Qzone communication can better explain the behaviour of human beings in network communication. However, few studies on the impact of emergencies and prediction methods based on social media data.

Research on human behaviour patterns based on mobile QQ spatial data. From the perspective of time interval, periodicity and fluctuation in the dynamics of human behaviour, the study analyses the patterns of human behaviour by comparing the posts and replies of QQ users across the country in case of emergencies [17]. Finally, analyze the trouble. By comparing different troubles and ordinary events, the network rules of other Posting methods are studied, and the behaviour rules of users are statistically analyzed based on the Posting and reply rules of users [18]. This paper provides a framework for analyzing QQ users' human behaviour patterns and social structure patterns and conducts empirical analysis based on QQ data to reveal the rules of QQ users' human behaviour patterns in the period of emergencies.

This paper has the following contributions. (1) Using mobile QQ space communication data, we dig out the statistical characteristics of human communication behaviour in the time dimension from the data of 5 emergencies and conventional events and the evolution pattern of human communication behaviour in the case of public security emergencies. (2) Explore the internal regularity of the impact of emergencies on human communication behaviour patterns and further predict human behaviour characteristics; And (3) the prediction of the impact degree of emergencies and the analysis and classification of human social attributes have potential application value.

The paper is organized as follows: Section 2 discusses related work, Section 3 presents the experimental results, and Section 4 concludes the article.

\section{Related work}

With the widespread application of social media, human social behaviours can be perceived in real-time, which provides a data basis for studying human behaviour patterns under emergencies and makes it possible to analyze the impact of crises quantitatively. Many scholars have carried out exploratory research on the quantitative analysis of the impact of emergencies based on the research on the dynamics of human behaviour under normal conditions.

\subsection{Research on temporal and spatial characteristics of human communication behaviour}

The time-domain model analysis of communication behaviour is to study the statistical law of human communication behaviour in time. The main statistical indexes include interval time, waiting time, time-series feature diversity analysis, array generation coefficient and memory coefficient, and array generation cycle division and statistics under different thresholds. Such as the number of events distributed within the occurrence cycle, the interval time of the array occurrence cycle and the waiting time of the array occurrence cycle. 
Interval time or wait time analysis is important to study the internal regularity of the occurrence of events. After Barab'asi discovered non-Poisson statistical properties of human email communication behaviour [6], the same phenomenon has been found in many other empirical results of human communication behaviour. Most of the results show that it can be fitted by power-law distribution [19]or exponentially truncated power-law distribution [20] at the population level. Still, different empirical results are also presented in some literature. The fitted distribution functions include lognormal distribution [21] and complex three-segment power-law[22] , etc. At the individual level, in addition to obeying power-law distribution [23], it also includes power-law plus exponential two-segment distribution[24], multi-segment power-law distribution [25], Weibull distribution [20], gamma distribution [26] and cascading Poisson process fitting [27].

Based on the empirical analysis data set of email, Barab'asi proposed the original task priority queue model [6], thus explaining most of the event processing process in daily human life regarding task deadlines and waiting time. The model assumes that human task execution strategies can be divided into three types: first-in, firstout, arbitrary execution, and highest priority execution. Simulation results confirm that the third strategy can generate task execution time series with power-law attenuation intervals. Subsequent scholars gradually improved this model, gave its exact solution and extended it [28]. Wu et al. used this model to reproduce the power-law plus exponential two-segment function characteristics of mobile phone SMS interaction [25].

From the above empirical research results of human spatial behaviour, it can be seen that using social media data to study the spatial pattern of human behaviour is an effective means, which is of great significance to understanding the law of human spatial movement.

\subsection{Research on behaviour rules in emergencies}

The researchers also used many other types of social media data to conduct in-depth analyses of the impact of emergencies, including Analyzing the impact of Hurricane Sandy on social communication based on Flicker data [29] or Twitter data [30] and analyzing the impact of Boston Marathon bombing on social communication based on Twitter data[31]. This paper analyzes sports by using the data of various network media, a presidential election or big storms and other mass incidents caused by the impact on the social communication [32], using GPS data analysis of the nuclear accident impact on personnel emergency evacuation behaviour [16], use of flight 911, volcanic eruption events such as data analysis of the impact of human movement mode [33], etc. In terms of emergency detection, Candia et al. studied the Spatiotemporal regularity of group communication behaviour by using the communication data of 6 million mobile phone users in a European country for one month and provided a quantitative method for the activity and fluctuation of call volume. These research methods have potential application value in emergency detection. Altshuler et al. proposed a method to detect emergencies based on social network data [33]. Based on simulation experiments, various types of emergency information given by Bagrow et al. [33] were used to verify the real network. Traag et al. analyzed the movement track information formed by about 900 million SMS messages and call 
records of 5.75 million mobile phone users in a European country over 14 months. The detection and participant identification method of social group gathering events based on the Bayesian location reasoning framework is presented [15] . Baker et al. analyzed mobile phone users' three-month travel trajectory data in Los Angeles, San Francisco, and New York. They gave the results of regional participation in a mass event, etc., pointing out that mobile phone data has a broad application prospect in the fields of mobile computing, urban planning, social-ecological analysis and epidemic transmission [34] .

\section{Methods}

\subsection{Data set}

In the monopoly era of mobile Qzone communication, the data of mobile Qzone communication can better explain the behaviour of human beings in network communication. However, few studies on the impact of emergencies and prediction methods based on social media data. According to the latest user tracking data, in October 2012, the average daily coverage of Tencent QQ reached 180 million people, and the arrival rate of Internet users reached 66.9 per cent. Alitwangwang has 37.47 million daily subscribers and 13.8 Internet users. The average daily coverage of Fexin.com reached 16.95 million people, ranking third with 6.3 of Internet users.

See Table 1,source: home office version, October 2012, based on the long-term monitoring data of 400000 homes and offices (excluding public Internet access places) Xiangben's network behaviour.

The average daily arrival rate of Internet users is the quotient of the average daily coverage of all websites compared to the total daily coverage.

According to the latest data from user tracker, in October 2012, Tencent QQ was used for 3.3 billion hours, accounting for 87.1 of the total time, ranking first among instant messaging software. Ali Want Want's effective use time reached 200 million hours, accounting for 5.4 of adequate use time. The effective use time of skew reached 69.77 million hours, accounting for 1.8 of the sufficient use time, and the three together accounted for 94.3 of the total adequate use time.The effective usage time rate is shown in Table 2.

The data in Table 2 are from the long-term monitoring data of online behavior of 400,000 households and offices (excluding public Internet access places) in October 2012.

The monthly useful time ratio in Table 2 is the ratio of the monthly useful time of the software to the sum of the monthly useful time of all the software in this category.

As mentioned above, in 2012, people's communication behaviour patterns and online opinion expression tools were relatively simple. Since Tencent QQ in 1999, QQ has gradually evolved into the most important and commonly used communication and chat software.

Similarly, when a particular event happens, people mainly publish some events in mobile QQ space to get friends' comments to know friends' views on the event. Therefore, we choose mobile QQ space data to conduct a comparative analysis of emergencies and routine events over the years in the social media data. Since mobile Qzone was the main way for people to express their opinions on the Internet, our 
research can verify different events and people's group behaviours through a single communication behaviour (Posting and reply on mobile Qzone).

In this chapter, we analyze and compare the time interval of Posting comments in mobile QQ space of four different types of emergencies and one typical event and conclude the influence of emergencies on human communication behaviour patterns.

\subsection{Statistical description of data}

The data analyzed in this paper are from the mobile QQ space data of 571 volunteers, including the complete data of 468 friends' mobile QQ space after removing invalid users. The period is from July 2005 to August 2014. As the most successful instant chat software in China and the related application of QQ, mobile Qzone also has a large number of active users. People can post messages, logs, and other interactive replies in mobile Qzone. And every behaviour of the user in the space (active initiator, passive initiator, time, content) will be recorded. In data cleaning, the crawling program is used to simulate the mobile QQ space of the browser login users for information crawling. This paper mainly analyses the time interval of the three typical Posting behaviours of the users in the mobile QQ space: talk, log and message. The accurate time of the data can be real to the second.The data details are shown in Table 3.

In data cleaning, we remove some abnormal data; for example, the time is 197001-01 00:00:00 or null. In the actual experiment, we try to make statistics on the time interval in days, hours, minutes and seconds, respectively. The experimental results show that Fitting time in seconds is more consistent with people's normal work and rest time, so we choose seconds as the unit for time interval analysis.

\subsection{Model of task priority queue}

Baraba'si et al. proposed and improved the task priority queue-based model, which uses task queues to explain most event processing in daily human life. They believe that tasks are limited by the deadline, and they need to arrange their work schedule according to the deadline and waiting time of functions. For a task in a queue, the length of its waiting time depends on the policy that people choose and execute it, and there are three main types:

IThe most straightforward execution strategy is the first-in, first-out approach, common in many service processes. The waiting time is the sum of the execution time of all previous charges. If the execution time of each lesson is a bounded distribution, the wait time distribution will form an exponential tail, indicating that the wait time of most tasks is almost the same.

IIThe random execution policy randomly selects tasks to be executed next regardless of task priorities and waiting time in queues. The distribution of task wait time is also exponential.

(III)Performs the task of top priority strategy is the most common method in human activities, at this point, the high-priority study shortly after joining the queue soon implemented, and low priority task requires geometric its high priority of tasks are performed to be executed, may wait a long time, perhaps this is observed in human behaviour characteristics of the thick tail.

At each time step of the model, the task with the highest priority is executed with probability $\mathrm{P}$, and a task is randomly selected with probability $1-P$. At this 
point, when $P ß 1$, the model describes strategy (III), and the distribution of waiting time predicted by the model presents a power-law feature with a power index of -1 . When $p ß 0$, the model describes the strategy (II), and the distribution of waiting time predicted by the model shows exponential decay.

We use the task priority queue model to analyze and explain the impact of emergencies on human behaviour.

\subsection{Paroxysmal and periodicity of human communication behaviour in emergencies} The (M, B) phase diagram used here can quantify the memorization and the paroxysmal of complex systems such as human behaviour and natural phenomena. The horizontal and vertical coordinates of the phase diagram are the memory coefficient (M) and the matrix coefficient (B), respectively. The memory coefficient M consists of the interval time series $\tau_{i}(i=1,2, \ldots)$ It is derived from the autocorrelation function, which is defined as:

$$
M=\frac{1}{n-1} \sum_{i=1}^{n-1} \frac{\left(\tau_{i}-m_{1}\right)\left(\tau_{i+1}-m_{2}\right)}{\sigma_{1} \sigma_{2}}
$$

Where $\mathrm{n}$ is the number of the time interval between mobile QQ space posts, $m 1(m 2)$ and $\sigma_{1}\left(\sigma_{2}\right)$ is comment interval time series $\tau_{i}(\tau+1),(i=1, \ldots, n-1)$. The matrix emission coefficient $\mathrm{B}$ is derived from the variation coefficient of the alarm interval probability distribution of an individual, which is defined as:

$$
\mathrm{B}=\frac{\sigma^{\tau}-\mathrm{m}^{\tau}}{\sigma^{\tau}+\mathrm{m}^{\tau}}
$$

Where, $\mathrm{m}^{\tau}$ and $\sigma^{\tau}$ are the samples mean and standard deviation of mobile QQ space Posting interval, respectively. If $B<0$ When 0 and 1 are shown in the time series, the time series has a strong memory, which is the more predictable, if the $m=0$ is neutral, if $m<0$; And when we tend to be 1 , we say that the time series is anti-memory. If $B>0$, When the time series is more powerful, the time sequence is neutral, the time sequence is the Poisson process, the behaviour is random if $B<0$. 0 and the time series tends to be periodic when it tends to be 1 .

\section{Experimental results}

When analyzing the distribution of human Posting time intervals in case of emergencies, we chose the Hailstorm event in Gansu. We considered four kinds of Posting behaviour: posting time interval distribution, talk Posting time interval distribution, log Posting time interval distribution, and message Posting time interval distribution. Interval time is the interval between two posts, expressed by power rate and represents its probability distribution. Logarithmic binning method is used in the statistical analysis to observe the damping trend in the Logarithmic coordinate system and calculate the power exponent.

\subsection{Distribution of human Posting time interval in case of emergencies}

As can be seen from the figure 1, the distribution of all kinds of Posting time intervals presents the characteristics of the heavy tail to varying degrees, and several 
Posting behaviours show the phenomenon of the alternating long silent period and short active period. Compared with the existing research results, the results of our experiment are consistent with users' Posting behaviours on blogs and microblogs, mobile phone messaging and QQ chat, etc. The difference is that MsgFeed posts are more heavy-tailed. In contrast, msGFeed is mainly caused by the fact that when people are dealing with natural disasters, they respond. People in the area do rescue and relief work, and people outside the area donate supplies or help rather than a comment. Post a wish in msgFeed after resolving the event or providing assistance, so there is a significant heavy tail feature in the figure and a long quiet period. People post less in blogs and have a long silent period. This is because the blog is a summary made by the owner of mobile QQ space on a recent experience, such as the 2014 annual summary, etc. In an emergency situation, people focus on this one event and blog posts decrease relative to other content.

\subsection{Time interval distribution of human comments in case of emergencies}

The comment record and time interval of a different type of post reflect the impact of the corresponding post in an emergency to show the impact of various kinds of correspondence. The life cycle of talk, log, and the message is analyzed in seconds in the double logarithm coordinate system.

See Figure 2; compared with people Posting in mobile QQ space, comments are more active because Posting is an individual action, and comments are a many-toone action. Comments on MsgFeed show two power laws, the first steeper than the second, fitting the human model of user publishing based on user interest decay. When people saw others' posts on msgFeed about the natural disaster, they actively discussed it. Still, eventually, as time went by, people's interest in the event declined sharply at first, then slowly declined and finally calmed down. Blogs and Msgboards generally do not publish natural disasters (emergencies), so they are generally not affected by events.

\subsection{Empirical analysis of emergencies}

To reveal the impact of crises on human behaviour, we use mobile QQ space Posting time interval to conduct an analysis experiment. According to four types of emergencies and one routine event, we divided the data of mobile QQ space into five groups, as shown in Table 4:

\section{The impact of natural disasters on human behavior}

In the figure 3, X-axis represents the time interval of people's Posting in mobile QQ space, and Y-axis represents the probability of occurrence under the time interval of Posting on the $\mathrm{X}$-axis. Take natural disasters as an example. On May 10, a massive hailstorm in Min County, Gansu province, caused mountain floods, killing 37 people. Regarding the time distribution of mobile QQ space posts when natural disasters occur, the corresponding five-day time interval distribution shows a negative correlation in the power index value. We found in the figure that the activity on the day of hail occurrence in Gansu was relatively high compared with that on the two days before and after. Still, as time went by, the activity decreased significantly, and there were even longer and more periods of silence on the day of a natural disaster than on the other two days. The main reason is that when a natural disaster 
(emergency) occurs, people will pay more attention to it. People will first spread and communicate about the emergency on the Internet, and when they receive the information, they will spend a lot of time on disaster relief or donation. It can be seen from the figure that in the case of natural disasters, people will enter the state of rescue and relief as early as possible after disseminating relevant information. The interval of post time silence period starts earlier than that of ordinary events and ends later. There is an active discussion period during natural disasters, but as circumstances change, people will lose their attraction, there will be periods of silence, and eventually normalcy.

\section{The impact of sudden accidents and disasters on human behaviour}

In the figure 4,taking the fire of Guangdong underwear factory on December 5, 2012 , as an example, there is a negative correlation between the power index values of the five groups of users in the time distribution of mobile QQ space posts when accidents and disasters occur. Similar to natural disasters, there is a longer period of silence than normal. Unlike natural disasters, people will pay more attention to the things around them when the underwear factory fire occurs, which will cause intense social discussion. Therefore, December 5 is more active than the other two days, and people actively participate in comments and posts, so the time interval between people's posts is not very long. It can be seen that during the period of single network expression tool, people pay more attention to manufactured accidents around them and make more positive comments.

\section{Impact of public health events on human behaviour}

In the figure 4,taking the anthrax outbreak on August 13, 2009, as an example, we verified the emergency response of people in the network in different emergencies. It can be concluded from the figure that there is a negative correlation between the power index values of the five groups of users in the time distribution of mobile QQ space Posting when public health events occur. After the anthrax outbreak on August 13, there was no significant difference in the power rate of people's comments on social media. The possible reason was that there was not a lot of information interaction on that day due to the single source of information and the lag of information. But people responded more promptly to the news later on, especially on August 14 and 15. It can be seen that during the period of single communication tools, people are still more sensitive to public health events. Data are updated every moment during public health events; people also pay attention to the latest trends and use communication tools to understand the current situation more frequently. The tail of the approximate exponential type indicates that only a small number of posts have intervals of more than one day, which are likely to be the intervals between individuals in the data set experiencing different emergencies.

\section{Impact of social security incidents on human behaviour}

In the figure 5,taking the hijacking incident of June 29, 2012, as an example, the time distribution of mobile QQ space posts when social security incidents occurred, the corresponding five groups of users showed a negative correlation in the power index value. There was no significant difference in the power of people's comments on social media on July 22, the day of the hijacking. Still, there was a considerable difference on July 23 because social security incidents were generally not reported in detail on that day. The news media received accurate news through some channels and made a composite news report, which was declared the next day. People 
intensely discussed this event, so there was a lot of discussion on social platforms two days after the social security incident.

\section{The impact of ordinary events on human behaviour}

Here, we select the everyday events from July 23 to July 27, 2012 and the previous five days with emergencies for comparison. As shown in the figure 6 , it is found that the silence period of human beings is not very long in ordinary events, and there is no longer fluctuation following the human behaviour law of "long silence, short burst" in normal circumstances.

\subsection{Comparative analysis of human communication behaviour in emergencies}

As can be seen from this figure 7, the silent period of mobile QQ space Posting in the case of emergencies is longer than that of ordinary events. It may be a process of a human responding to emergencies. In the part of the array, the time interval of mobile QQ space Posting is closer than that of ordinary events, and it has the characteristics of the fat tail.

Taking all of these sudden events together, we have the following findings: In an emergency, the human behaviour of "long silence, short wave" has changed, and the silent period of human beings is longer. However, in the short term, in the case of emergencies, human Posting behaviour will be more frequent and intensive than usual.

When an emergency occurs, there will be an obvious heavy tail distribution. Mobile QQ space post time interval also with the passage of time and the formation of a power rate attenuation trend. Emergencies will affect the law of human behaviour laterally, and people will respond to it. When accidents and natural disasters occur, people will start the silent period of Posting in advance to help those affected by the disaster. Accidents or social security incidents will cause strong discussions after the event and eventually calm down.

\subsection{Paroxysmal and periodicity of human communication behaviour in emergencies}

This paper measures mobile QQ space Posting behaviour based on the (M, B) phase diagram and further verifies the suddenness and memorability of industrial communication social behaviour,(M, B) Please refer to Section 3.4 for details. Figure 8 shows the $(\mathrm{M}, \mathrm{B})$ phase diagram of individual Posting behavior sequence within the range of daily Posting quantity greater than 1 and less than 50 from 2006 to 2011. It can be seen that almost all values are in the first two quadrants of the phase diagram. Their mean values were $0607 M B=(0.060,0.211)$, $0809 M B=(0.148,0.284), 1011 M B=(0.157,0.229), 1213 M B=(0.104,0.284)$. As can be seen from the picture that the probability distribution of communication behaviour interval time is stronger than the exponential distribution of the Poisson process $(B 0)$ and $M=0.148$. Therefore, it can be concluded that communication behaviour's time series memory features to present a medium level. Figure 8 distribution of occurrence coefficient B of all individuals' alarm time sequence array, its peak appears at 0.8 , and the time series of mobile QQ space Posting behaviour of about 99 in the group is more than 0 . Such paroxysmal level is because individuals may send out spatial posts several times in a short period in the face of an emergency. In contrast, the interval between the two troubles is longer.FIG. 9 
shows the temporal memory coefficient $\mathrm{M}$ distribution of all individuals' mobile QQ spatial Posting behaviours, and its peak value appears at 0 . As can be seen from the picture that 83 of mobile QQ space Posting behaviour, time-series memory is above the medium level.

To compare and analyze the Posting behaviour characteristics of mobile QQQ space in years with more emergencies and years with fewer emergencies, fig. 3 shows the analysis results of several other real data sets. It includes mobile QQ space Posting time interval of 2006 and 2007 (red), mobile QQ space Posting time interval of 2008 and 2009 (blue), mobile QQ space Posting time interval of 2010 and 2011 (blue), mobile QQ space Posting time interval of 2012 and 2013 (plum red). As can be seen from the figure, in 2008 and 2009 and 2012 and 2013 (two years with more emergencies), the time series of mobile QQ space Posting behaviour showed stronger paroxysmal than other behaviours. But more special is the other four years of mobile QQ space paroxysmal data is higher. In addition, the mean value of memory is similar to the results of mobile QQ space Posting in 2010 and 2011 years because they have similar ways of dealing with emergencies. Still, certain differences in the urgency of crises and response demands drive users' Posting behaviour.

We selected all the emergencies in 2012 or 2009 when we studied the previous chapter. Then we decided the day and two days before and after these emergencies to make an analysis chart of paroxysmal and memory.

Figure 9 shows the (M, B) phase diagram of sudden and memorizable Posting behaviour in mobile QQ space under the above four kinds of emergencies. As can be seen from the figure, the mean point of the $(\mathrm{M}, \mathrm{B})$ phase diagram is $(-0.176,0.326)$, which has paroxysmal solid and weak anti-memory. According to empirical research, human behaviour is a strong, weak memory. However, under the influence of emergencies, the bursts of human Posting behaviour are more potent than those of ordinary events, and human behaviour even shows anti-memory. The strengthening of paroxysmal is because the emergence of emergencies will cause people to intensely discuss and conduct activities, resulting in abnormal human movements. The reason for the occurrence of anti-memory is that people choose different response measures by judging the degree of urgency and response demand in dealing with emergencies, unlike ordinary events, so that they will have a weak memory.

\subsection{Prediction of human Posting behaviour}

A prediction of human Posting behaviour can well analyze human behaviour under the influence of emergencies. In this chapter, 84,535 thousand mobile QQ space data of 468 QQ users in the first 10 months of 2009 are used for model training, and 25131 mobile QQ space data of QQ users in the last two months are used for accuracy verification. Through the data analysis of 4 or 8 users, it is found that there will be a noticeable trend of increasing the number of users' posts when there is an emergency.

With the decrease of the heat of the event, the number of users' posts per week will return to about 2000. The verification results showed that the ACF and PACF graphs were not stable, which could not explain the accuracy of the prediction. As you can see from the picture below that not only the amount of data Posting has some discrepancy, but also the trend of human Posting in the mobile QQ space has 
a severe deviation. It may be because of the uncertainty and instability caused by emergencies and the weak anti-memory of human behaviour in emergencies that the prediction can not be realized.

In figure 11, the analysis and prediction chart of QQ space Posting volume, the lighter coloured line represents the training set, the darker black line represents the verification set, and the red line represents the prediction made through the training set.

In figure 12 we can see that the autocorrelation graph shows first-order hysteresis, but the autocorrelation value does not exceed the boundary value. Although the autocorrelation value of order 0 exceeds the boundary in the figure, it may be accidental that the autocorrelation value does not exceed the standard in other orders. From this, we can conclude that our prediction parameters can be used.

And figure 13 slants in the 4 orders are the first time since the correlation over boundary values we selected here q 4 through the analysis of the data of 468 users found that in case of emergency, humans will have an apparent increase trend of postings, as events heat drop, we have weekly postings back to about 2000 users. The verification results showed that the ACF and PACF graphs were not stable, which could not explain the accuracy of the prediction. It can be seen from the figure that not only the amount of data Posting has some discrepancy, but also the trend of human Posting in the mobile QQ space has a severe deviation. It may be because of the uncertainty and instability caused by emergencies and the weak anti-memory of human behaviour in emergencies that the prediction can not be realized.

\section{Results and Discussion}

With the development and progress of Internet technology, people's communication on social networks is widespread, replacing previous communication methods such as letters, emails, and short messages. Therefore, the research on the Posting behaviour and comment behaviour of mobile QQ space can understand the temporal characteristics of human dynamics under the influence of emergencies from one side. In this paper, 273,522 posts and 920,619 comment interaction records were counted. The time interval between users' posts and replies under troubles was analyzed, and the outbreaks and memorability of different emergencies to human beings and Posting behaviours.

It also makes a predictive analysis of human Posting behaviour. It is found that the time interval between people's Posting behaviour and interactive comment behaviour in mobile QQ space can be described by a power-law distribution in case of emergency. The time interval distribution of post and reply is an apparent heavytail distribution with a long silent period forms a trend of power rate attenuation with time. Finally, it becomes calm with the resolution of emergencies. In terms of paroxysmal, human beings will have more assertive paroxysmal communication behaviour in the face of emergencies than before. What's remarkable here is that when it comes to memory, humans even can resist it. The final failure to predict human Posting behaviour is due to the different stress responses of human beings in the face of various emergencies. These findings provide evidence and reference for future studies to truly understand the characteristics of human behaviour under the influence of emergencies. 
Funding

This work was supported in part by the Key RD program of Zhejiang Province (2022C01083), The National Science Foundation of China (62102262)and Zhejiang Province Public Welfare Technology Application Research Project (LGG18F020012).

\section{Abbreviations}

Not applicable.

Availability of data and materials

The datasets used and/or analyzed during the current study are available from the corresponding author on reasonable request.

Ethics approval and consent to participate

Not applicable.

Competing interests

The authors declare that they have no known competing financial interests or personal relationships that could have appeared to influence the work reported in this paper.

Consent for publication

All authors agreed to publish.

Authors' contributions

Xiaolin Wei: Mainly responsible for data collating and writing.Zhenzhen Chen: Mainly modified and polished the article.Yi Luo: Design paper framework and model. Hongyan Li and Xuming Zhao : Primarily designed the thesis rogram. Tao Wang and Zhenhuan Wu: Getting data and the results verified.

Author details

${ }^{1}$ Zhejiang Yuexiu University, Shaoxing, China. ${ }^{2}$ School of International Business, Zhejiang Yuexiu University Shaoxing, China. ${ }^{3}$ Shaoxing Key Laboratory for Smart Society Monitoring, Prevention control, Shaoxing, China. ${ }^{4}$ Library, Zhejiang Yuexiu University, Shaoxing, China. ${ }^{5}$ Department of Computer Science and Engineering,

Shaoxing University, Shaoxing, China. ${ }^{6}$ College of Economic and Management, Zhejiang University of Science and Technology, Hangzhou, China. ${ }^{7}$ UCSI Graduate Business School, UCSI University, Kuala Lumpur, Malaysia.

References

1. Barabási, A.: Scale-free networks: A decade and beyond. Science $\mathbf{3 2 5}$

2. Social science. computational social science. Science (2009)

3. Castellano, C., Fortunato, S., Loreto, V.: Statistical physics of social dynamics. Review of Modern Physics 81(2) (2007)

4. Manifesto of computational social science. The European Physical Journal Special Topics 214(1), 325-346 (2012)

5. Pentland, A.: Social physics : how good ideas spread-the lessons from a new science. Research-Technology Management 58(6188), 1097-1097 (2015)

6. Si, A.: The origin of bursts and heavy tails in human dynamics

7. Brockmann, D., Hufnagel, L., Geisel, T.: The scaling laws of human travel. Nature 439(7075), $462-465$ (2006)

8. Oliveira, J.G., Barabási, A.: Darwin and einstein correspondence patterns. Nature

9. Malmgren, R.D., Stouffer, D.B., Campanharo, A., Amaral, L.: On universality in human correspondence activity. Science 325(5948), 1696-1700 (2009)

10. Iribarren, J.L., Moro, E.: Impact of human activity patterns on the dynamics of information diffusion. Physical Review Letters 103(3), 038702 (2009)

11. Goh, K.I., Barabasi, A.L.: Burstiness and memory in complex systems. arXiv e-prints (2006)

12. Girardin, F., Calabrese, F., Fiore, F.D., Ratti, C., Blat, J.: Digital footprinting: Uncovering tourists with user-generated content. IEEE Pervasive Computing 7(4), 36-43 (2008)

13. Watts, D.: Characterizing individual communication patterns (2015)

14. Song, X., Zhang, Q., Sekimoto, Y., Shibasaki, R.: Prediction of Human Emergency Behavior and Their Mobility Following Large-scale Disaster. Prediction of human emergency behavior and their mobility following large-scale disaster, ??? (2014)

15. Traag, V.A., Browet, A., Calabrese, F., Morlot, F.: Social event detection in massive mobile phone data using probabilistic location inference. In: IEEE Third International Conference on Privacy (2012)

16. Altshuler, Y., Fire, M., Shmueli..., E.: The social amplifier-reaction of human communities to emergencies. Journal of Statistical Physics 152(3), 399-418 (2013)

17. Yang, G., Yin, Y., Hong, M.: Human object interactions recognition based on social network analysis. In Applied Imagery Pattern Recognition Workshop (2014)

18. Li, J., Liu, Y., Liu, H., Wang, B., Wang, L.: Analysis of the influence of individual web behavior pattern on group behavior simulation. In: 2019 IEEE Fourth International Conference on Data Science in Cyberspace (DSC) (2019)

19. Kan, A., Chan, J., Hayes, C., Hogan, B., Bailey, J., Leckie, C.: A time decoupling approach for studying forum dynamics (2012)

20. Jiang, Z.Q., Xie, W.J., Li, M.X., Podobnik, B., Zhou, W.X., Stanley, H.E.: Calling patterns in human communication dynamics. Proc Natl Acad Sci U S A 110(5), 1600-1605 (2013)

21. Stouffer, D.B., Malmgren, R.D., Amaral, L.: Log-normal statistics in e-mail communication patterns. Physics 53(06), 187-225 (2006) 
22. Chun, H., Kwak, H., Eom, Y.H., Ahn, Y.Y., Jeong, H.: Comparison of online social relations in terms of volume vs. interaction: A case study of cyworld. In: Proceedings of the 8th ACM SIGCOMM Conference on Internet Measurement 2008, Vouliagmeni, Greece, October 20-22, 2008 (2008)

23. Nnl, A., Ning, Z.A., Tao, Z.: Empirical analysis on temporal statistics of human correspondence patterns sciencedirect. Physica A: Statistical Mechanics and its Applications 387(25), 6391-6394 (2008)

24. Bza, B., Jw, A.: Topography analysis of particle velocity distribution function in gas-solid flow. Chemical Engineering Science 197, 69-73 (2019)

25. Peng, W., Lei, T., Chi, H.Y., Wang, B.H.: Heterogenous human dynamics in intra and inter-day time scale. EPL (Europhysics Letters) 94, 18005 (2011)

26. J., -L., Guo, C., Fan, Z., -H., Guo: Weblog patterns and human dynamics with decreasing interest. The European Physical Journal B 81(3), 341-344 (2011)

27. Anteneodo, C., Malmgren, R.D., Chialvo, D.R.: Poissonian bursts in e-mail correspondence. European Physical Journal B 75(3), 389-394 (2010)

28. Gibbs, J.W.: Elementary principles in statistical mechanics. Elementary Principles in Statistical Mechanics ii(1), 55-59 (2014)

29. Preis, T., Moat, H.S., Bishop, S.R., Treleaven, P., Stanley, H.E.: Quantifying the digital traces of hurricane sandy on flickr. Scientific Reports 3, 3141 (2013)

30. Wang, Q., Taylor, J.E.: Quantifying human mobility perturbation and resilience in hurricane sandy. Plos One 9 (2014)

31. Lin, Y.R., Margolin, D.: The ripple of fear, sympathy and solidarity during the boston bombings. EPJ Data Science 3(1), 1-28 (2014)

32. Szell, M., Grauwin, S., Ratti, C.: Contraction of online response to major events. Plos One 9 (2014)

33. Bagrow, J.P., Wang, D., Barabási, A., Moreno, Y.: Collective response of human populations to large-scale emergencies. Plos One 6(3), 17680 (2011)

34. Becker, R., Caceres, R., Hanson, K., Isaacman, S., Loh, J.M., Martonosi, M., Rowland, J., Urbanek, S., Varshavsky, A., Volinsky, C.: Human mobility characterization from cellular network data. Communications of the ACM 56(1), 7476-82 (2013)

Figures
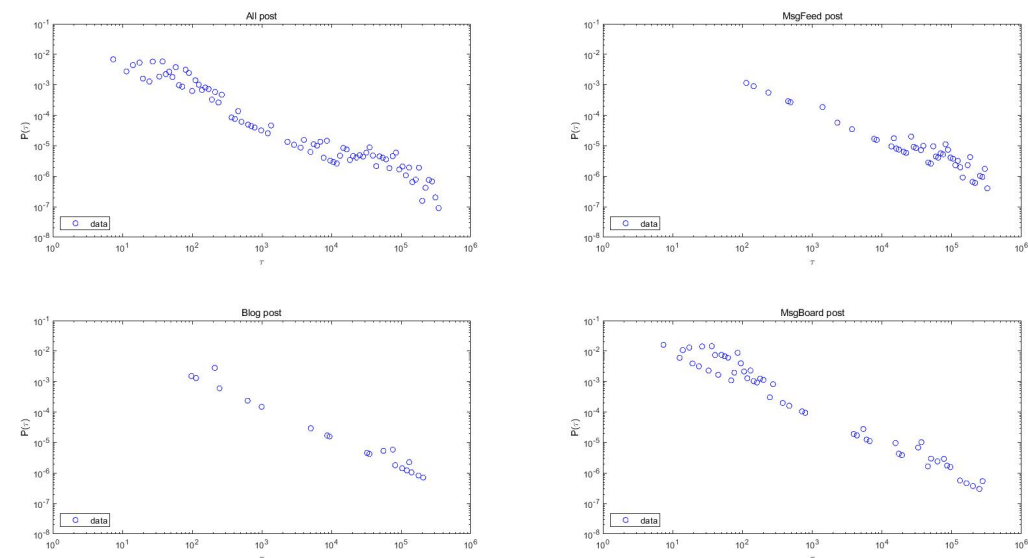

Figure 1 Distribution of human Posting time interval in case of emergencies

Tables

Table 1 Daily coverage of real communication software in October 2012

\begin{tabular}{cccc}
\hline Ranking & Software & Average daily coverage(ten thousand people) & Rate of Internet Users \\
\hline 1 & Tencent QQ & 18103 & 66.9 \\
2 & Ali Wangwang & 3747 & 13.8 \\
3 & Feixin & 1695 & 13.8 \\
4 & Crooked & 741 & 2.7 \\
5 & MSN & 645 & 2.4 \\
6 & Renren desktop & 491 & 1.8 \\
7 & Microblog deskto & 318 & 1.2 \\
8 & Tencent RTX & 254 & 0.9 \\
9 & Tencent TM & 211 & 0.8 \\
10 & Skype & 179 & 0.7 \\
\hline
\end{tabular}



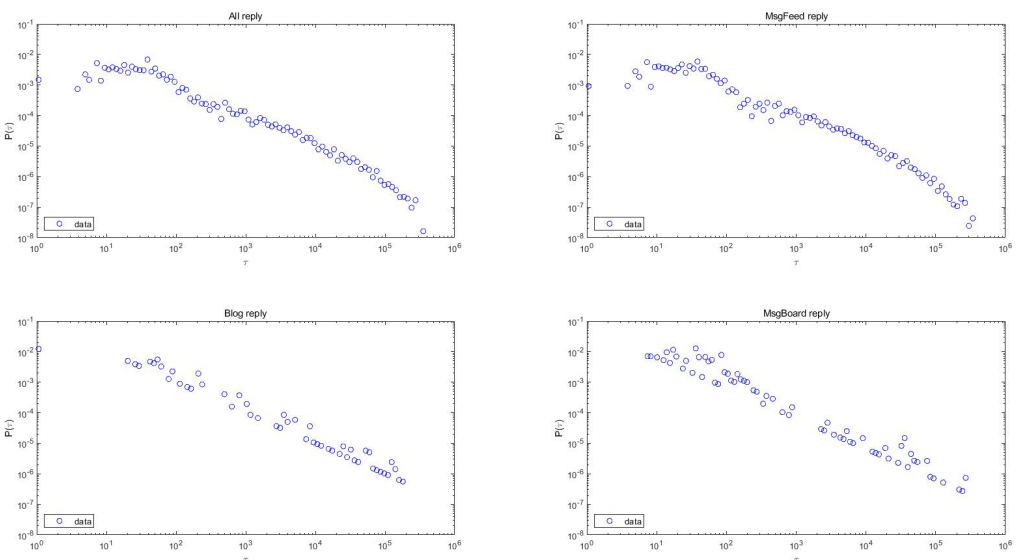

Figure 2 Time interval distribution of human comments in case of emergencies

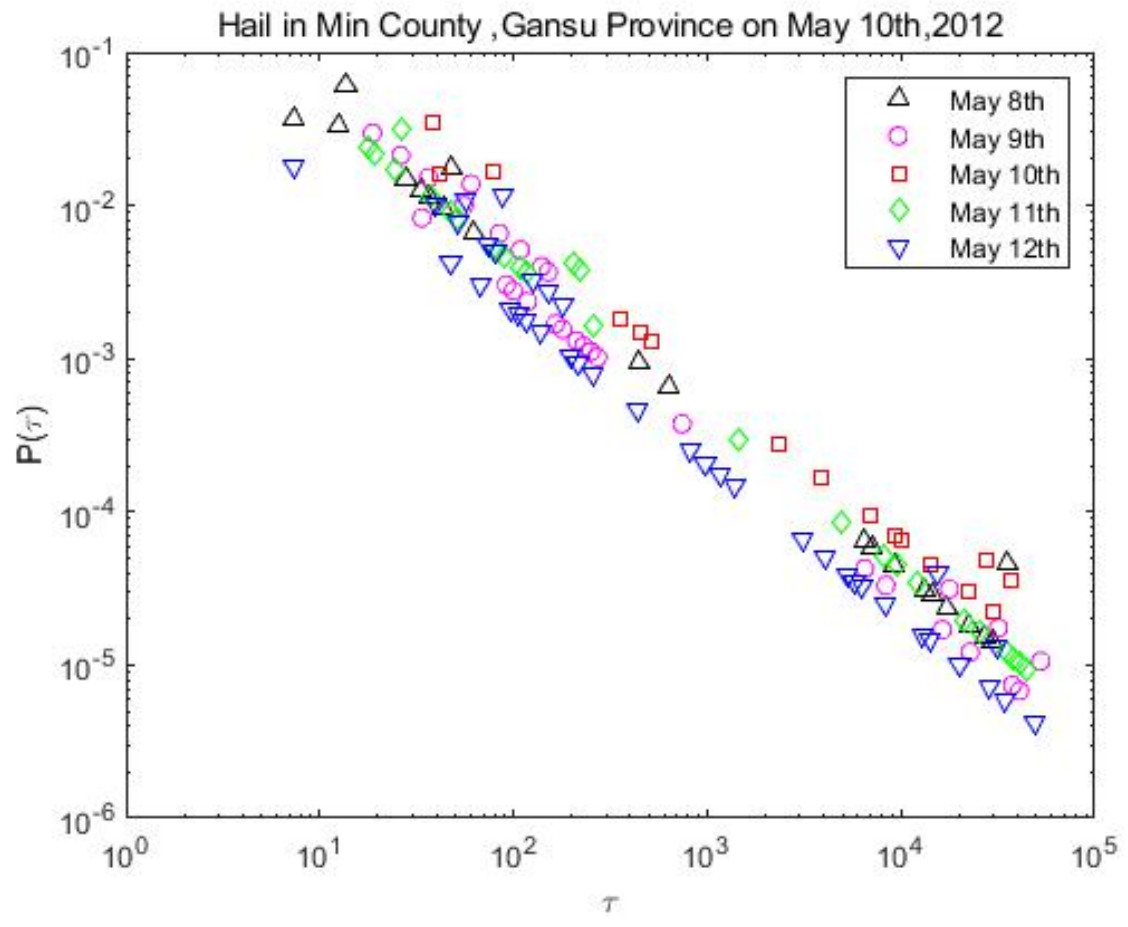

Figure 3 Distribution of human post time interval under the influence of accident disaster 


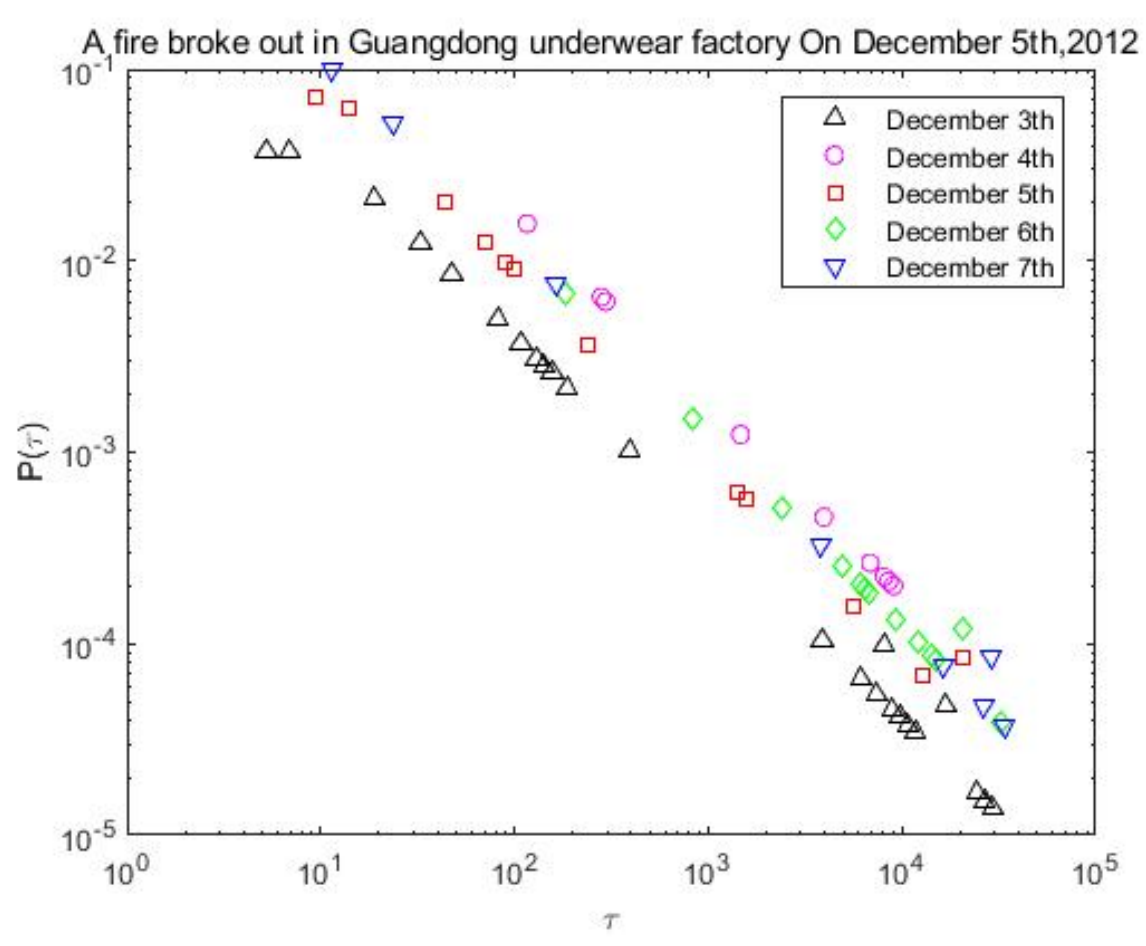

Figure 4 Distribution of human post time intervals under the influence of public health events

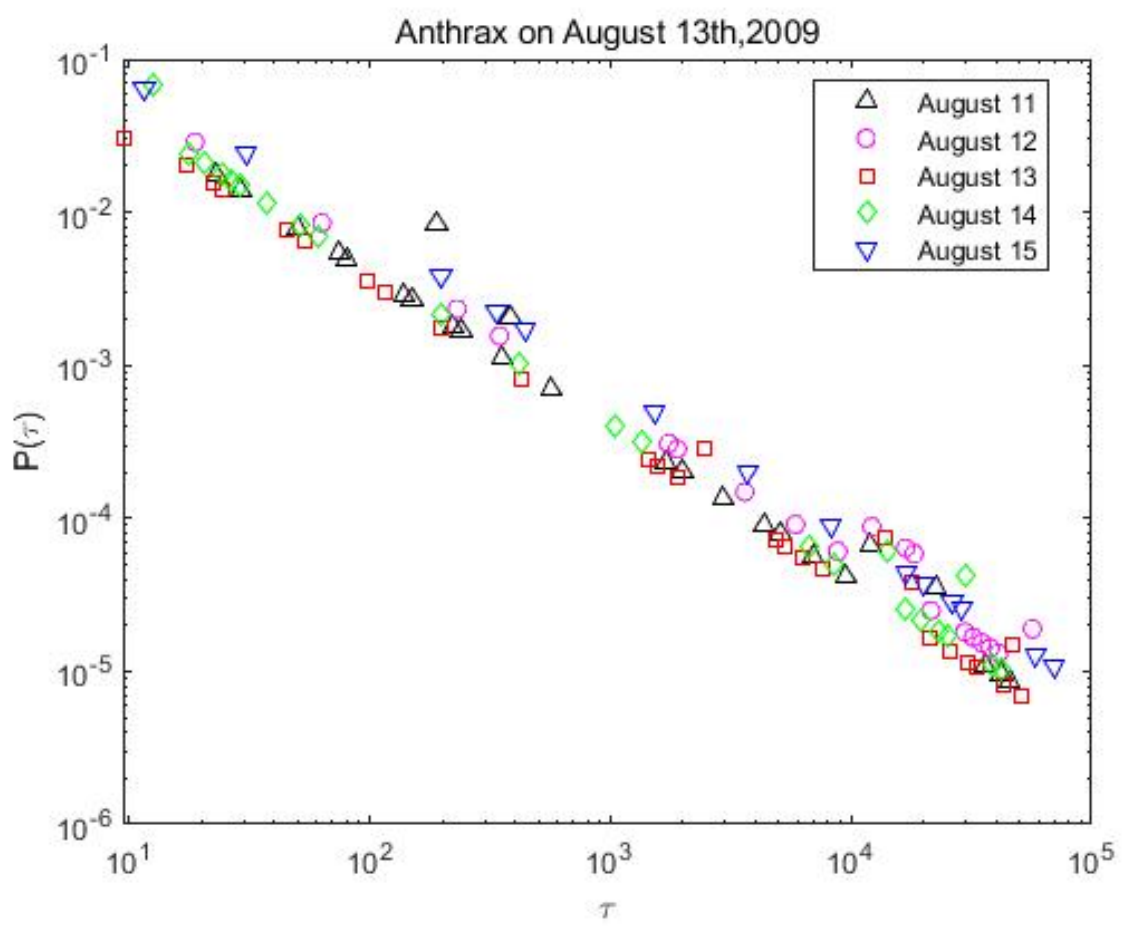

Figure 5 Distribution of human Posting time interval under the influence of social security events 

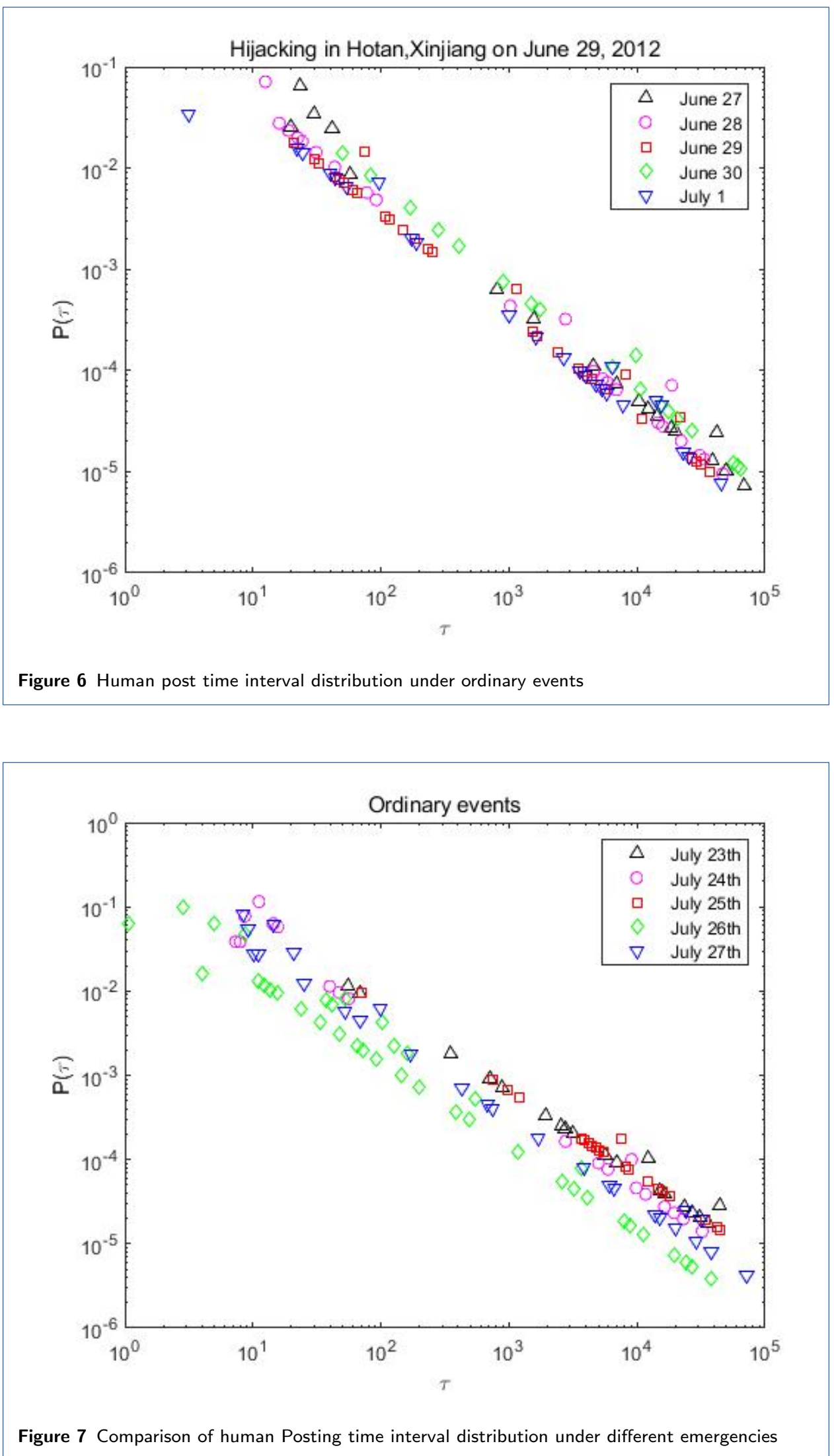


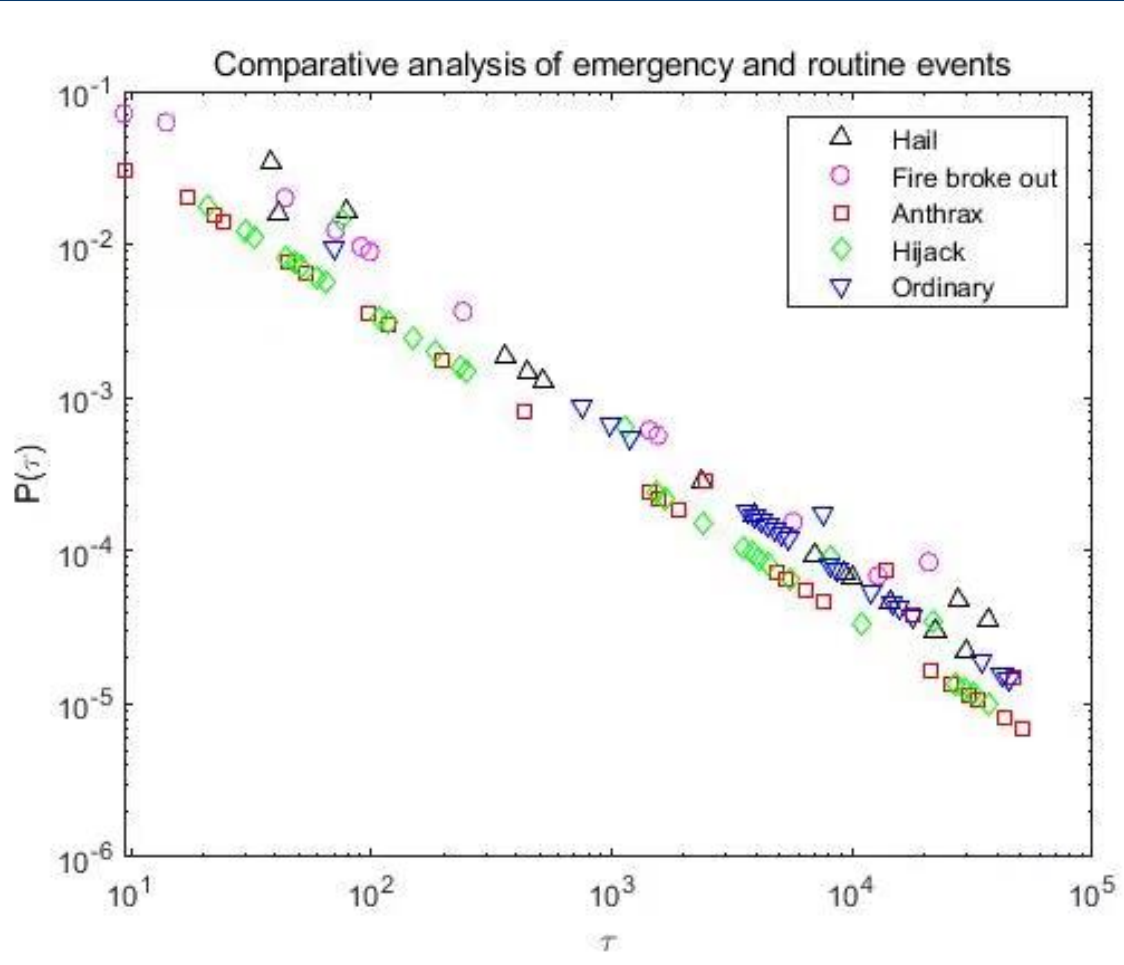

Figure 8 Paroxysmal and memorability of QQ space posts from 2006 to 2014

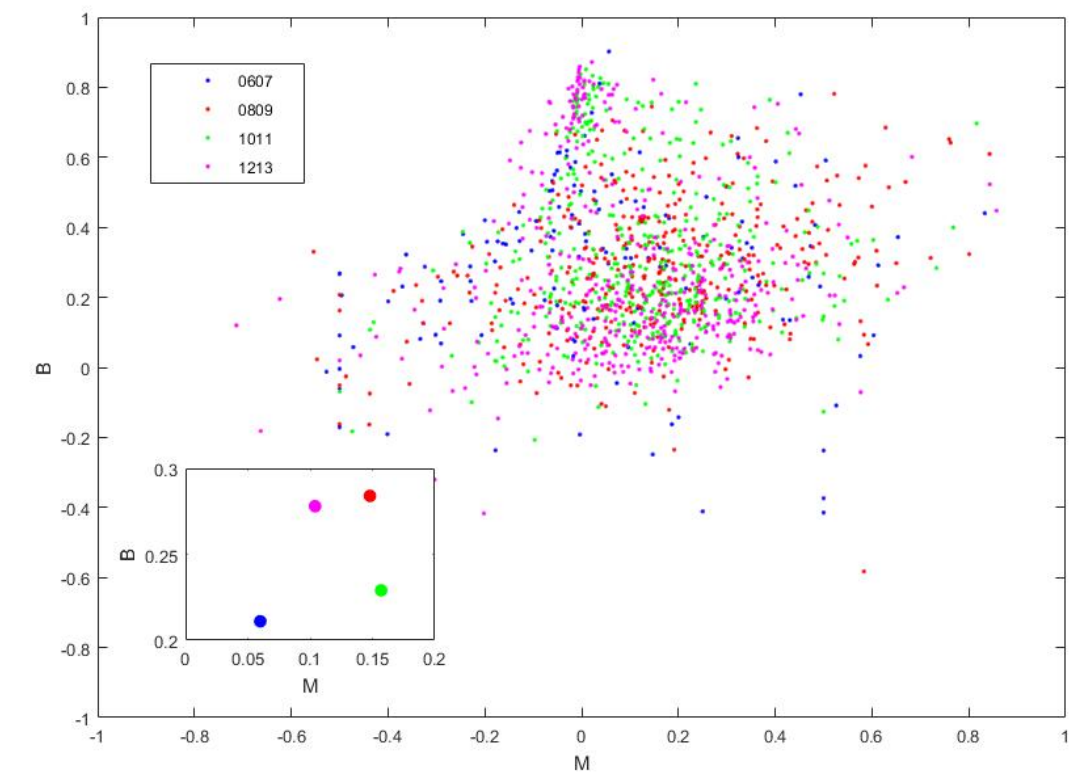

Figure 9 Paroxysmal and memorability of emergencies 


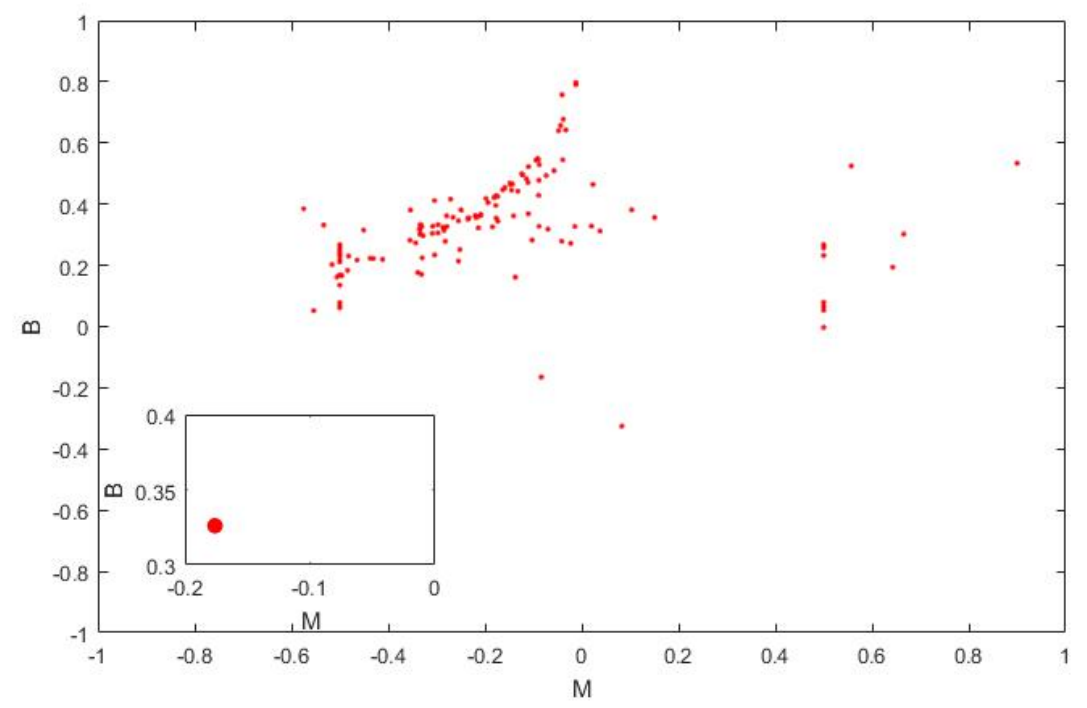

Figure 10 Analysis and prediction of $Q Q$ space post volume

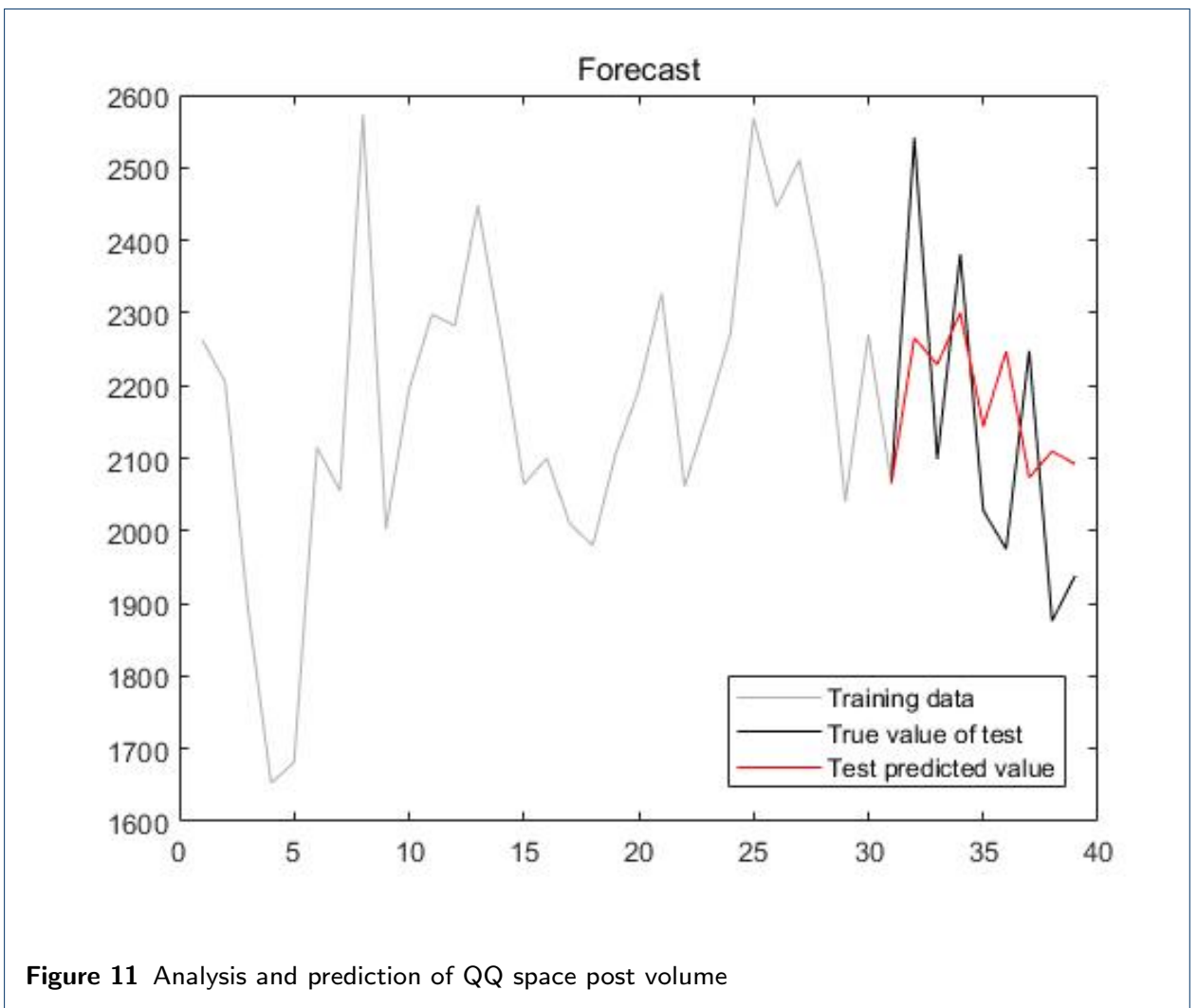




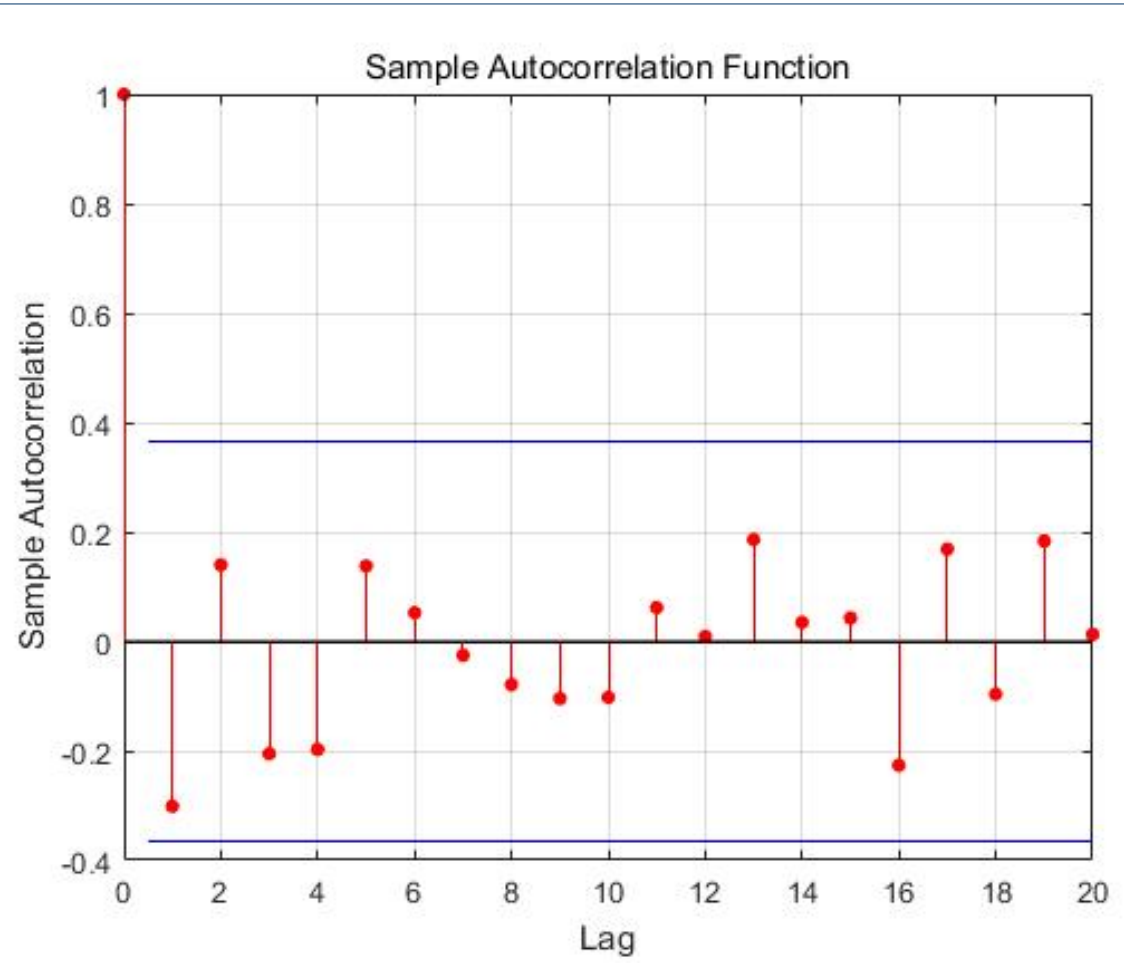

Figure 12 Analysis and prediction of QQ space post volume

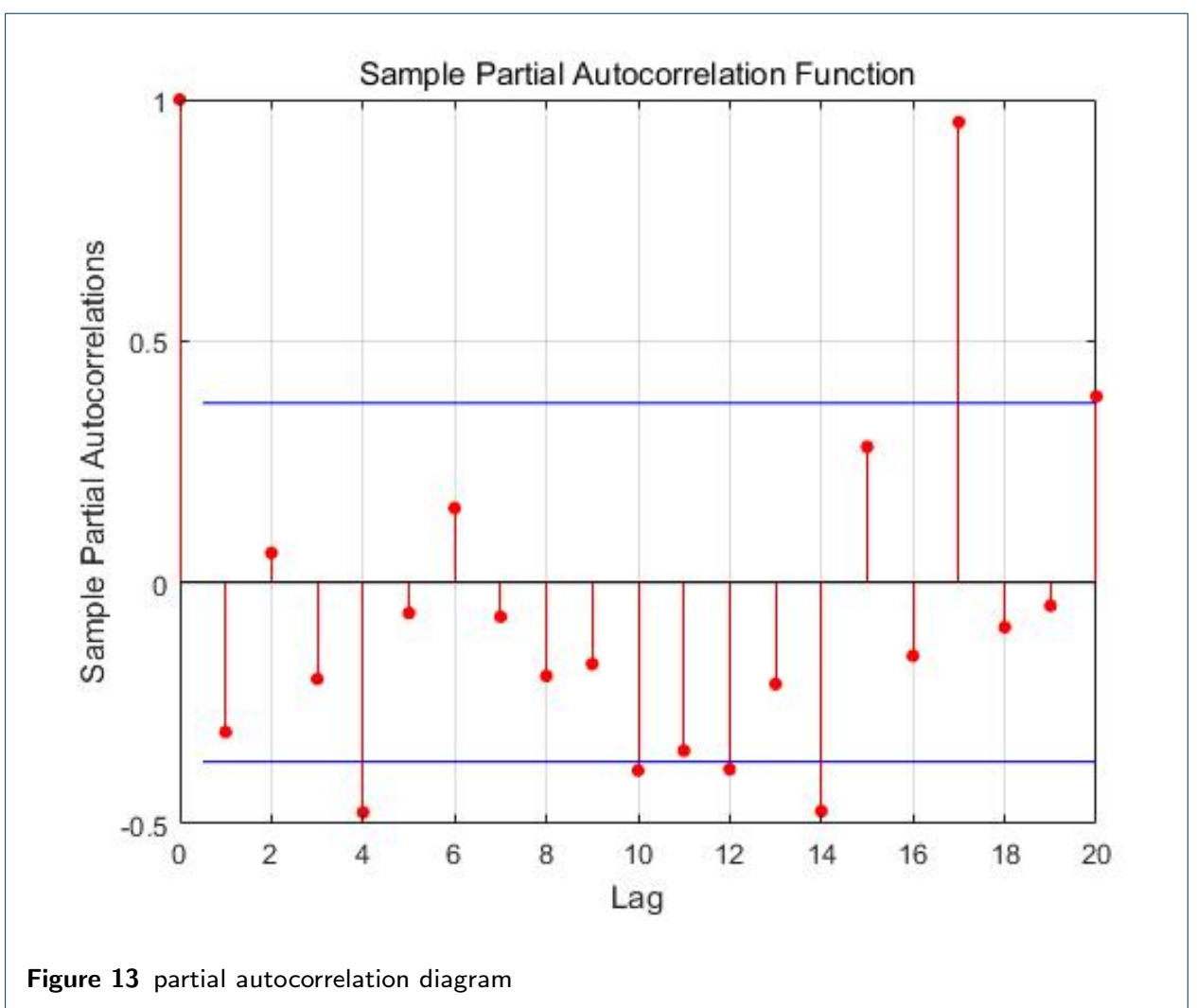


Table 2 Statistical table of daily active use events of real-time communication software in October 2012

\begin{tabular}{cccc}
\hline Ranking & Software & Monthly effective use time & Monthly effective use time \\
\hline 1 & Tencent QQ & 330258 & 87.1 \\
2 & Ali Wangwang & 20341 & 5.4 \\
3 & Feixin & 6977 & 1.8 \\
4 & Crooked & 6210 & 1.6 \\
5 & MSN & 2806 & 0.7 \\
6 & Renren desktop & 2540 & 0.7 \\
7 & Microblog deskto & 2350 & 0.6 \\
8 & Tencent RTX & 2276 & 0.6 \\
9 & Tencent TM & 1821 & 0.5 \\
10 & Skype & 1234 & 0.3 \\
\hline
\end{tabular}

Table 3 Data summary table

\begin{tabular}{ccccc}
\hline Type & Log & Message & Board & Summary \\
\hline Post & 140996 & 40809 & 91599 & 273404 \\
Reply & 630111 & 202790 & 87718 & 920619 \\
\hline
\end{tabular}

Table 4 Event grouping table

\begin{tabular}{|c|c|c|c|c|c|}
\hline Category & Group 1 & Group 2 & Group 3 & Group 4 & Group 5 \\
\hline Event type & Natural disasters & Disaster & Public health & Social security & Ordinary events \\
\hline Event & Hail & Fire & Anthrax & Hijacking & Events \\
\hline At the time of incident & 282 & 254 & 121 & 429 & 333 \\
\hline The day before the incident & 230 & 202 & 156 & 350 & 310 \\
\hline Two days before the event & 381 & 261 & 152 & 361 & 265 \\
\hline One day after the incident & 356 & 253 & 135 & 285 & 484 \\
\hline Two days after the event & 316 & 267 & 129 & 280 & 300 \\
\hline
\end{tabular}

